\title{
Determination of Ferret Enteric Coronavirus Genome in Laboratory Ferrets
}

\author{
Tian-Cheng Li, Sayaka Yoshizaki, \\ Michiyo Kataoka, Yen Hai Doan, Yasushi Ami, \\ Yuriko Suzaki, Tomofumi Nakamura, \\ Naokazu Takeda, Takaji Wakita
}

Ferret enteric coronavirus (FRECV) RNA was detected in laboratory ferrets. Analysis of the complete genome sequence of 2 strains, FRCoV4370 and FRCoV063, revealed that FRECV shared $49.9 \%-68.9 \%$ nucleotide sequence identity with known coronaviruses. These results suggest that FRECV might be classified as a new species in the genus Alphacoronavirus.

$\mathrm{F}$ erret coronavirus (FRCoV), a novel animal coronavirus $(\mathrm{CoV})$, was identified in ferrets (Mustela putorius furo) in 2006 (1). However, only partial sequences of FRCoV have been analyzed, including portions of open reading frame (ORF) $1 \mathrm{~b}$ and the full-length spike protein $(\mathrm{S})$, nonstructural protein $3 \mathrm{c}(3 \mathrm{c})$, envelope protein $(\mathrm{E})$, membrane protein $(\mathrm{M})$, nucleocapsid protein $(\mathrm{N})$, and accessory genes ( $3 \mathrm{x}$ and $7 \mathrm{~b}$ ), and only in 3 strains: 1 ferret systemic coronavirus (FRSCV), FRSCV MSU-1 strain (GenBank accession no. GU338456); and 2 ferret enteric coronaviruses (FRECVs), FRECV MSU-2 strain (GenBank accession no. GU338457) and FRECV No22 strain (GenBank accession no. LC029419) (2,3). Genetic analyses based on these partial sequences showed that FRCoV is closer to mink coronavirus (MCoV) than to other CoVs and appears to be a member of the genus Alphacoronavirus in the subfamily Coronovirinae, which also contains the genera Betacoronavirus and Gammacoronavirus (4). To further understand the constellation of FRCoVs, we analyzed the complete genome.

\section{The Study}

In our previous study, we detected ferret hepatitis $\mathrm{E}$ virus (HEV) in fecal samples from laboratory ferrets and confirmed that $40(63.5 \%)$ of the 63 ferrets were infected with ferret HEV (5). For the observation of the ferret HEV

Author affiliations: National Institute of Infectious Diseases, Tokyo, Japan (T.-C. Li, S. Yoshizaki, M. Kataoka, Y.H. Doan, Y. Ami, Y. Suzaki, T. Wakita); Research Foundation for Microbial Diseases of Osaka University, Kagawa, Japan (T. Nakamura); Osaka University, Osaka, Japan (N. Takeda)

DOI: https://doi.org/10.3201/eid2309.160215 particles by electron microscopy, we used a $10 \%$ suspension prepared from the fecal specimen from 1 of the ferrets (no. F4370), concentrated by ultracentrifugation and purified by sucrose gradient ultracentrifugation. However, instead of finding ferret HEV particles, we observed many coronavirus-like particles in fractions 4,5 , and 6 , with densities of $1.230 \mathrm{~g} / \mathrm{cm}^{3}, 1.214 \mathrm{~g} / \mathrm{cm}^{3}$, and $1.198 \mathrm{~g} / \mathrm{cm}^{3}$ (data not shown). These particles ranged from $60 \mathrm{~nm}$ to $120 \mathrm{~nm}$ in diameter; most of them had a spike structure, suggesting that the ferrets were infected with a CoV-like virus.

To precisely examine these $\mathrm{CoV}$-like particles, we diluted 63 fecal specimens with 10-mmol/L PBS for preparation of the $10 \%$ suspension. We extracted the RNA by using a MagNA Pure LC Total Nucleic Acid isolation kit (Roche Applied Science, Mannheim, Germany) according to the manufacturer's recommendations. We performed an FRSCV-specific reverse transcription PCR (RT-PCR) with the primer set G1F (5'-CTGGTGTTTGTGCAACATCTAC- $\left.3^{\prime}\right)$ and G1R (5'-TCTATTTGCACAAAATCAGACA-3') and an FRECV-specific RT-PCR with the primer set G2F (5'-GGCATTTGTTTTGATAACGTTG-3') and G2R (5'-CTATTAATTCGCACGAAATCTGC-3') (2).

RT-PCR results revealed that, of the 63 ferret fecal specimens, 22 (34.9\%) were positive for FRSCV RNA, 53 (84.1\%) were positive for FRECV RNA, and 15 (23.8\%) were positive for both FRSCV and FRECV RNA. The specimen from ferret F4370 was positive only for FRECV RNA. These results indicated that the ferrets were infected extensively with FRCoV. However, we observed no signs such as weight loss or diarrhea in the ferrets.

We extracted RNA from a pool of fractions 4,5 , and 6 from the F4370 fecal sample suspension and analyzed the complete genome sequence of FRCoV by using a next-generation sequence analysis (6). FRCoV4370 (GenBank accession no. LC119077) has a genome size, gene order, genomic organization, and structure similar to those of known alphacoronaviruses. The complete genome of FRCoV4370 contains 28,525 nt and a poly (A) tail. Except for the 5'-terminus $261 \mathrm{nt}$ and 3 '-terminus $247 \mathrm{nt}$ untranslated regions, FRCoV4370 encodes 9 proteins: ORF1a, ORF1a/1b, S, 3c, $\mathrm{E}, \mathrm{M}, \mathrm{N}, 3 \mathrm{x}$, and $7 \mathrm{~b}$ (Table). Although the ORF7a protein was found in the MCoVs WD1127 (GenBank accession no. HM245925) and WD1133 (accession no. HM245926), we did not find it in FRCoV4370.

The complete genome of FRCoV4370 shared $49.9 \%-68.9 \%$ nucleotide identity with other known CoVs. 
Table. Nucleotide positions of proteins of FrCoVs from analysis of complete virus genome and number of amino acids compared with $\mathrm{MCoVs}^{*}$

\begin{tabular}{|c|c|c|c|c|c|c|c|c|c|}
\hline \multirow[b]{2}{*}{ Proteins } & \multicolumn{2}{|c|}{ Nucleotide position } & \multicolumn{7}{|c|}{ No. amino acids } \\
\hline & FRCoV4370 & FRCoV063 & FRCoV4370 & FRCoV063 & MSU-2 & MSU-1 & No22 & WD1127 & WD1133 \\
\hline ORF1a & $262-12228$ & $262-12231$ & 3,988 & 3,989 & NA & NA & NA & 4,018 & 4,006 \\
\hline ORF1a/b & 262-20222 & 262-20225 & 6,653 & 6,554 & NA & NA & NA & 6,682 & 6,670 \\
\hline Spike & 20215-24564 & 20218-24567 & 1,449 & 1,449 & 1,449 & 1,457 & 1,435 & 1,438 & 1,429 \\
\hline ORF3c & 24585-25328 & 24585-25328 & 247 & 247 & 247 & 83 & 247 & 247 & 69 \\
\hline E & $25297-25545$ & 25297-25545 & 82 & 82 & 82 & 82 & 82 & 82 & 82 \\
\hline M & 25560-26351 & 25560-26363 & 263 & 267 & 263 & 263 & 265 & 268 & 268 \\
\hline $\mathrm{N}$ & 26368-27492 & 26380-27504 & 374 & 374 & 374 & 374 & 374 & 376 & 376 \\
\hline ORF7a & & & $\mathrm{NI}$ & $\mathrm{NI}$ & $\mathrm{NI}$ & $\mathrm{NI}$ & $\mathrm{NI}$ & 98 & 98 \\
\hline ORF3x & $27501-27725$ & 27514-27738 & 74 & 74 & 74 & 14 & 74 & 73 & 73 \\
\hline ORF7b & $27664-28278$ & $27677-28291$ & 204 & 204 & 204 & 204 & 202 & 204 & 204 \\
\hline
\end{tabular}

${ }^{*} \mathrm{E}$, envelope protein; FrCOV, ferret coronavirus; M, membrane protein; MCoV, mink coronavirus; N, nucleocapsid protein; NI, protein not identified; NA, sequence not available; ORF, open reading frame.

Phylogenetic trees based on the complete genome demonstrated that FRCoV4370 is closer to MCoVs than to other $\mathrm{CoVs}$, although it clearly separated into a distinct cluster (Figure, panel A). We observed similar phylogenetic clustering when we compared $\approx 8,300 \mathrm{nt}$ sequences of the 3 '-termini of CoVs. FRCoV4370 forms a new cluster with FRSCV MSU-1, FRECV MSU-2, and No22, being separated from the cluster formed by MCoVs (Figure, panel B). In addition, BLAST analysis (http://blast.ncbi.nlm.nih.gov/Blast.cgi) using the nucleotide sequences described previously revealed that FRCoV4370 shared 94\% identity with FRECV MSU-2, which is higher than that of FRSCV MSU-1 (89\%), indicating that FRCoV4370 belongs to the FRECVs.

As is the case for other CoVs, 2 long ORFs were predicted in the FRCoV4370 genome: ORF1a contains 11,967 nt, from nt 262 to 12228 , encoding 3,988 aa; ORF1b encodes 7,830 nt, from nt 12393 to 20222 . The coronaviruses have a pseudoknot tertiary structure that allows a ribosomal shift of the reading frame between ORF1a and ORF1b (7-9). We also found the slippery sequence for the ribosomal shift (UUUAAAC) in the FRCoV4370 genome at nt positions 12192-12198. The ribosomal shift may have resulted in generation of the ORF1a/b protein encoding $19,962 \mathrm{nt}$ and the deduced 6,653 aa.

We identified 4 structural proteins in FRCoV4370: S, $\mathrm{E}, \mathrm{M}$, and $\mathrm{N}$. The $\mathrm{S}$ protein is the largest, consisting of 1,449 aa, which shared $92.5 \%$ aa identity with FRECV MSU2, 81.4\% with FRSCV MSU-1, and $84.5 \%$ with FRECV No22. The BLAST analysis of the $\mathrm{S}$ protein showed that it had low aa identities $(43 \%-66 \%)$ with other CoVs.
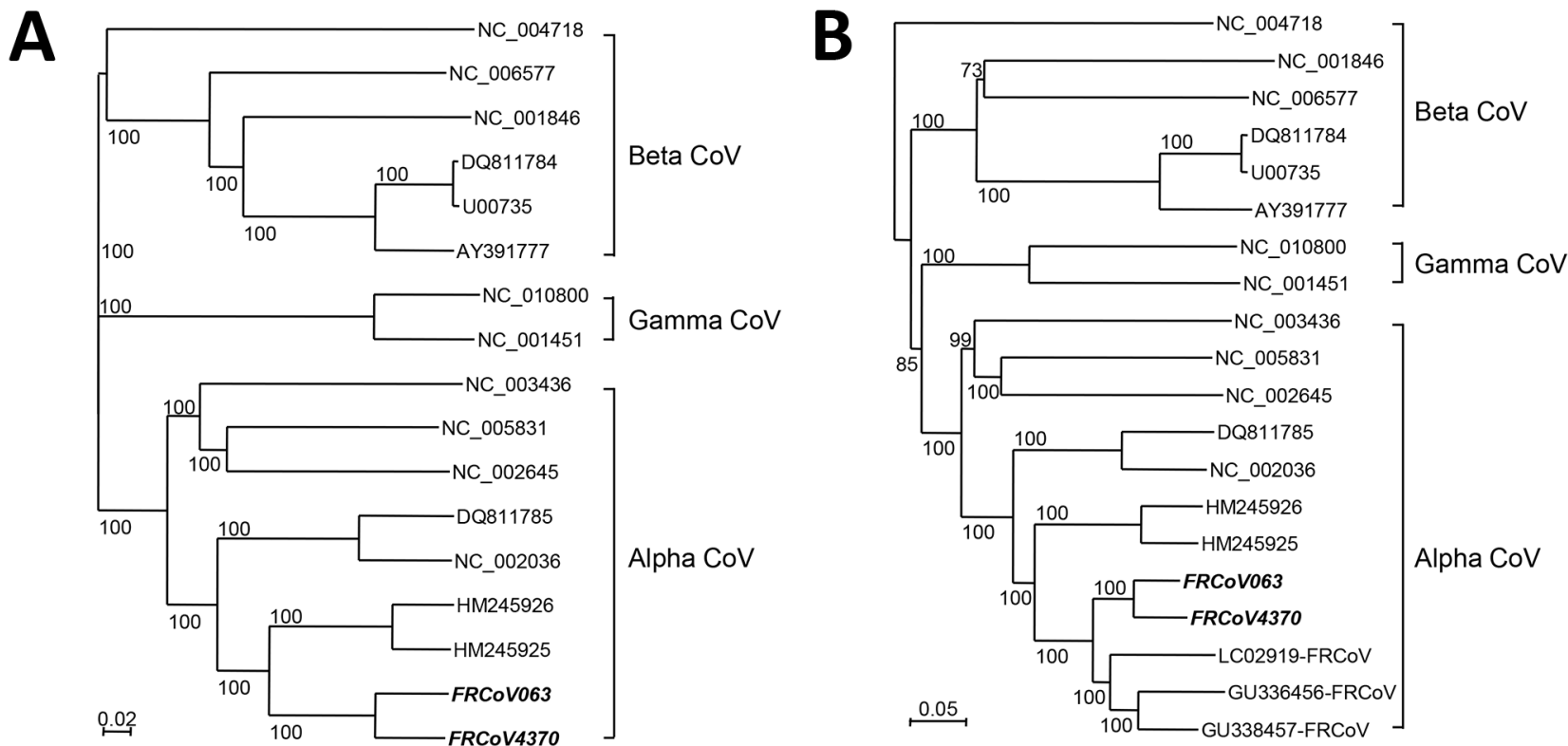

Figure. Phylogenetic relationships between ferret coronaviruses (FrCoVs, shown in bold italics) and other known coronaviruses (CoVs). A) Complete genome; B) partial 3'-terminus genome. The nucleic acid sequence alignment was performed using ClustalX version 1.81 (http://www.clustal.org). The genetic distance was calculated by Kimura's 2-parameter method. Phylogenetic trees with 1,000 bootstrap replicates were generated by the neighbor-joining method (Njplot 2.3, http://njplot.sharewarejunction.com/). Comparison CoVs identified by GenBank accession number. Scale bars indicate substitutions per site. 
Because the S protein is a major inducer of virus-neutralizing antibodies, the antigenicity and serotype of FRCoVs might be different from those of other CoVs.

The E protein, the smallest structural protein, encodes 82 aa (which is the same number encoded by other known FRCoVs) and shared $94.8 \%$ aa identity with FRECV MSU2, 85.6\% with FRSCV MSU-1, and $86.7 \%$ with FRECV No22. The 263 -aa M protein shared $81.6 \%$ aa identity with FRECV MSU-2. The low aa identity between the FRECV strains suggests that the membrane gene is highly variable. The N protein of FRCoV4370 contains 374 aa, making it the shortest among the alphacoronaviruses. The $\mathrm{N}$ protein shared $96.3 \%$ aa identity with FRECV MSU-2, 93.4\% with FRSCV MSU-1, and $80.2 \%$ with FRECV No22.

ORF3c was identified in FRCoV4370, as observed in MCoVs. This protein is an accessory triple-spanning membrane protein and is analogous to SARS-CoV $3 \mathrm{a}$ protein (10). The predicted ORF3c protein contains 247 aa and shared $96.8 \%$ aa identity with FRECV MSU-2 and $77.6 \%$ with FRECV No22. The 2 other nonstructural proteins, $3 \mathrm{x}$ and $7 \mathrm{~b}$, are located downstream of the $\mathrm{N}$ protein and encode 74 aa and 204 aa, respectively.

We also detected FRECV RNA in the fecal specimens of 9 ferrets from another farm in the United States and analyzed the complete genome of 1 strain, FRCoV063 (LC215971). The genome of FRCoV063 shared 94.0\% nucleotide identity with FRCoV4370 and had a similar structure (Table; Figure), suggesting that ferret coronavirus infection is common in ferrets, and genetically similar FRCoV strains circulate at the ferret farms.

\section{Conclusions}

Since the initial identification of FRCoV in ferrets in 2006, many sequences of FRSCV and FRECV strains have been analyzed (1-3,11-15). However, the complete genome sequences have not been determined and added to the public databases. In this study, we successfully analyzed the complete genome of 2 strains of FRECV, FRCoV4370 and FRCoV063, and found that they shared $94.0 \%$ nucleotide identity with each other but $49.9 \%-68.9 \%$ nucleotide identities with other known CoVs, suggesting that the ferret coronaviruses might be classified as a new species in the genus Alphacoronavirus. This new knowledge of the complete genome sequence of FRECV will contribute to investigations of the diversity of animal CoVs and will help establish new taxonomic units.

This study was supported by a Grant-in-Aid for Scientific Research (C) from the Ministry of Education, Culture, Sports, and Science, Japan.

$\mathrm{Dr} . \mathrm{Li}$ is a senior researcher at the National Institute of Infectious Diseases in Tokyo, Japan. His research focuses on epidemiology, the expression of viral proteins, and advances in the diagnosis of and vaccines for hepatitis $\mathrm{E}$ virus.

\section{References}

1. Wise AG, Kiupel M, Maes RK. Molecular characterization of a novel coronavirus associated with epizootic catarrhal enteritis (ECE) in ferrets. Virology. 2006;349:164-74. http://dx.doi.org/ 10.1016/j.virol.2006.01.031

2. Wise AG, Kiupel M, Garner MM, Clark AK, Maes RK. Comparative sequence analysis of the distal one-third of the genomes of a systemic and an enteric ferret coronavirus. Virus Res. 2010;149:42-50. http://dx.doi.org/10.1016/j.virusres.2009.12.011

3. Terada Y, Minami S, Noguchi K, Mahmoud HY, Shimoda H, Mochizuki M, et al. Genetic characterization of coronaviruses from domestic ferrets, Japan. Emerg Infect Dis. 2014;20:284-7. http://dx.doi.org/10.3201/eid2002.130543

4. Vlasova AN, Halpin R, Wang S, Ghedin E, Spiro DJ, Saif LJ. Molecular characterization of a new species in the genus Alphacoronavirus associated with mink epizootic catarrhal gastroenteritis. J Gen Virol. 2011;92:1369-79. http://dx.doi.org/ 10.1099/vir.0.025353-0

5. Li TC, Yang T, Ami Y, Suzaki Y, Shirakura M, Kishida N, et al. Complete genome of hepatitis $\mathrm{E}$ virus from laboratory ferrets. Emerg Infect Dis. 2014;20:709-12. http://dx.doi.org/10.3201/ eid2004.131815

6. Li TC, Yang T, Yoshizaki S, Ami Y, Suzaki Y, Ishii K, et al. Construction and characterization of an infectious cDNA clone of rat hepatitis E virus. J Gen Virol. 2015;96:1320-7. http://dx.doi.org/10.1099/vir.0.000072

7. Brierley I, Boursnell ME, Binns MM, Bilimoria B, Blok VC, Brown TD, et al. An efficient ribosomal frame-shifting signal in the polymerase-encoding region of the coronavirus IBV. EMBO J. 1987;6:3779-85.

8. Brierley I, Digard P, Inglis SC. Characterization of an efficient coronavirus ribosomal frameshifting signal: requirement for an RNA pseudoknot. Cell. 1989;57:537-47. http://dx.doi.org/ 10.1016/0092-8674(89)90124-4

9. Thiel V, Ivanov KA, Putics A, Hertzig T, Schelle B, Bayer S, et al. Mechanisms and enzymes involved in SARS coronavirus genome expression. J Gen Virol. 2003;84:2305-15. http://dx.doi.org/10.1099/vir.0.19424-0

10. Oostra M, de Haan CA, de Groot RJ, Rottier PJ. Glycosylation of the severe acute respiratory syndrome coronavirus triple-spanning membrane proteins 3a and M. J Virol. 2006;80:2326-36. http://dx.doi.org/10.1128/JVI.80.5.2326-2336.2006

11. Dominguez E, Novellas R, Moya A, Espada Y, Martorell J. Abdominal radiographic and ultrasonographic findings in ferrets (Mustela putorius furo) with systemic coronavirus infection. Vet Rec. 2011;169:231. http://dx.doi.org/10.1136/vr.d4705

12. Garner MM, Ramsell K, Morera N, Juan-Sallés C, Jiménez J, Ardiaca M, et al. Clinicopathologic features of a systemic coronavirus-associated disease resembling feline infectious peritonitis in the domestic ferret (Mustela putorius). Vet Pathol. 2008;45:236-46. http://dx.doi.org/10.1354/vp.45-2-236

13. Lescano J, Quevedo M, Gonzales-Viera O, Luna L, Keel MK, Gregori F. First case of systemic coronavirus infection in a domestic ferret (Mustela putorius furo) in Peru. Transbound Emerg Dis. 2015;62:581-5. http://dx.doi.org/10.1111/tbed.12407

14. Murray J, Kiupel M, Maes RK. Ferret coronavirus-associated diseases. Vet Clin North Am Exot Anim Pract. 2010;13:543-60. http://dx.doi.org/10.1016/j.cvex.2010.05.010

15. Provacia LB, Smits SL, Martina BE, Raj VS, Doel PV, Amerongen $\mathrm{GV}$, et al. Enteric coronavirus in ferrets, the Netherlands. Emerg Infect Dis. 2011;17:1570-1.

Address for correspondence: Tian-Cheng Li, Department of Virology II, National Institute of Infectious Diseases, 4-7-1 Gakuen, Musashi-murayama, Tokyo 208-0011, Japan; email: litc@nih.go.jp 